\title{
Subject Index to Volume 25
}

Acetaldehyde, disposition, during late pregnancy, rat, 102

Acetylcholine, cerebral vascular responses, ischemia effect, piglet, 180

Acidemia, propionate, long-chain fatty acids in, 147

Acidosis

lactic, fatal, with complex III defect, neonate, 553

metabolic, acyl-coenzyme A dehydrogenase deficiency, mice, 38

Aciduria

dicarboxylic, acyl-coenzyme A dehydrogenase deficiency, mice, 38

methylmalonic, long-chain fatty acids in, 147

Acyl-coenzyme A dehydrogenase, shortchain, deficiency, mice, 38

Adenosine monophosphate, cyclic, formation, children with pertussis, 209

Adenosine triphosphate

depletion, after oxidant injury, endothelial cell, 97

effect of oxygen exposure, bronchoalveolar fluid, rat, 396

Adolescent, growth status, zinc and copper deficiency and, 323

$\beta$-Adrenergic activity, myocardial oxygen consumption and, during hypoxemia neonatal lamb, 585

$\alpha$-Adrenoceptor, postjunctional responses, regional variation, sheep, 461

Age, serum mucins and, cystic fibrosis, 49

Airway, immature, in vivo mechanical properties, preterm lamb, 143

Albumin, vascular to alveolar leak, ventilated lung, immature rabbit, 130

Aldosterone, neonatal fluid homeostasis, 591

Alkalosis, effect on hypoxia-induced pulmonary vasoconstriction, neonatal rabbit, 383

Amino acid

branched chain

maple syrup urine disease, 11

maternal phenylketonuria treated with, rat, 568

Ammonium chloride, palmitic acid oxidation and, rat liver, 119

Amniotic fluid, dilution studies, perinatal baboon, 377

Androgen, renin processing and, submandibular gland, mouse, 332

Anemia, neonatal, prevention method, rabbit, 274

Anticoagulation, heparin and, neonatal plasma, 405

Antigen, mucin-associated levels, cystic fibrosis, 49

Antioxidant, developmental biology, piglet intestine, 612

Apolipoprotein, plasma, gastric drip feeding and, glycogen storage disease, 327

Arousal response, carotid-denervation and,

Artery developing hypoxemia, lamb, 473

intrapulmonary, response to vasoactive compounds, lamb, 360

wall properties, noninvasive assessment, 94
Ataxia, $B_{1}$ variant of $\mathrm{G}_{\mathrm{M} 2}$ gangliosidosis, 89

Atrial natriuretic factor

physiology, fetus and newborn, rat, 339

renal hemodynamic response, fetal and newborn sheep, 291

Atrial natriuretic peptide, neonatal fluid hemostasis, 591

Bacteria, opsonization, immune globulin intravenous effect, premature serum 634

Bilirubin

${ }^{125}$ I-albumin-bound, blood brain barrier permeability for, neonatal piglet, 452

intestinal absorption, saccharolactone effect, in presence of human breast milk, rat, 234

toxicity

delayed effects, neuroblastoma cell line, 369

neuroblastoma cell line, 364

Birth weight, insulin-like growth factor-I and thyroxine relationship, 336

Blood, rheologic properties, comparison, adult and neonate, 457

Blood brain barrier, permeability, neonatal piglet, 452

Blood flow: see also Circulation

hepatic, changes at birth, sheep, 300

myocardial, hypoxemia effect, during exercise, dog, 280

ocular and cerebral, effect of arterial $\mathrm{PCO}_{2}$ variations, piglet, 205

Blood pressure, pulmonary, patent ductus arteriosus, neonatal lamb, 136

Blood sampling

dilution studies, perinatal baboon, 377

neonatal anemia and, rabbit, 274

Blood viscosity, reduction, Fahraeus-Lindqvist effect, 595

Body water, estimation, dilution kinetics of markers, perinatal baboon, 377

Brain

hypoxic-ischemic injury

flunarizine pretreatment, infant rat, 573

measurement, phosphorus magnetic resonance spectroscopy, neonate, 445

Breastfeeding

fatty acid synthesis during, high carbohydrate diet effect, 63

saccharolactone effect on bilirubin absorption, rat intestine, 234

vitamin D metabolism, infants and mothers, 623

Breathing cycle, periodic, duration, preterm infants, 258

Bronchoalveolar fluid, adenosine triphosphate content, oxygen exposure and, rat, 396

Bronchopulmonary dysplasia

dexamethasone effect, infant mice, 353

preterm rat model, 525

Calcium

absorption, fortified human milk, very low birth weight infants, 496

cardiopulmonary effects, persistent pulmonary hypertension, neonate, 262 mitochondrial transport, rat intestine, 107 placental clearance, growth-retarded rat fetus, 420

Calcium channel, development, gastric smooth muscle, rabbit, 600

Carbohydrate, hepatic metabolism, after um bilical cord compression, fetal lamb, 228

Carbon dioxide, pressure, arterial variations, effect on blood blow, neonatal piglet, 205

Carnitine, palmitic acid oxidation and, rat liver, 119

Carotid, denervation, effect on arousal response to hyoxemia, lamb, 473

Cartilage column, formation, costochondral junction, neonates and children, 202

Chemoreceptor, peripheral tone, during hypoxic exercise, 285

Chloralose, basal hemodynamics after, neonatal lamb, 389

Chloride-bicarbonate, exchange, maturation, rat ileum, 189

Cholera toxin, adenylate cyclase response, suckling rat, 225

Chondrocyte, somatomedin-C and growth hormone effect, bovine growth plate, 76

Circulation, nitroprusside effect, aortopulmonary shunt, lamb, 44

Colostrum

antioxidant heterogeneity, further characterization, 266

lymphocytes, immunoglobulin production by, 295

Complex I, deficiency, mitochondrial encephalomyopathy, 194

Complex III, fatal defect of, neonatal lactic acidosis, 553

Convulsions, $B_{1}$ variant of $\mathrm{G}_{\mathrm{M} 2}$ gangliosidosis, 89

Copper

deficiency, growth status and, 323

toxicity, vitamin $E$ effect, rat hepatocyte,

Corticosteroids, gestational effects, ventilated rabbit, 32

Costochondral junction, column formation in, neonates and children, 202

Cystic fibrosis

pancreatic growth and GP-2 content, reserpinized rat model, 478

reserpine treatment effect on pancreas morphology, rat model, 482

serum mucin levels, age and clinical status related to, 49

Dexamethasone

effect on chronic pulmonary oxygen toxicity, infant mice, 353

effect on vitamin K-dependent carboxylation, fetal rat lung, 530

Diabetes, maternal, lung maturation in macrosomic offspring, mouse, 173

Diaphragm, contractility, digoxin effect, piglet, 271

Diarrhea

neonatal, role of adenylate cyclase, rat, 225 
secretory, Klebsiella pneumoniae heat-stable enterotoxin, 514

Diazepam, neonatal seizures treated by, nuclear magnetic resonance spectroscopy, dog, 27

Diet: see also Nutrition

protein turnover and, human milk and formula-fed infants, 167

Digoxin, diaphragmatic contractility and, piglet, 271

Doppler system, multigate pulsed, artery wall assessment, 94

Ductus arteriosus

closure, after surfactant replacement therapy, preterm infants, 305

prenatal ligation

pulmonary hypertension and, neonatal lamb, 239

pulmonary vasculature remodeled by, lamb, 251

Endorphin, acetylated and shortened, fetal pituitary gland, 652

Endothelium, adenosine triphosphate depletion, after oxidant injury, 97

Energy, substrate utilization, during total parenteral nutrition, infants, 645

Enteroinsular axis, full-term neonates, 490

Enterotoxin, heat-stable, Klebsiella pneumoniae diarrhea, 514

Enzyme

galactose-metabolizing, hepatic, pregnant rat and fetus, 161

oxidant-producing, developmental biology, piglet intestine, 612

Epidermal growth factor

binding, intrauterine growth retardation, rat, 214

receptor/kinase, ontogeny, ovine lung, 535

Epidermis, structural proteins in, perinatal rat, 542

Epinephrine, pulmonary artery response to, fetal and neonatal lamb, 360

Erythrocyte, insulin-like growth factor I binding to, normal prepubertal children, 409

Erythropoietin, recombinant radiolabeled, placental permeability, sheep, 649

Ethanol, disposition, during late pregnancy, rat, 102

Euglycemic hyperinsulinemic clamp, developmental response, neonatal dog, 219

Exercise

myocardial blood flow during, hypoxemia effect, dog, 280

ventilatory control during, hypoxia effect, 285

Fahraeus-Lindqvist effect, blood viscosity reduction by, neonates and adults, 595

Fasting, catch-up growth after, glucocorticoid-treated rats, 373

Fat, utilization, during total parenteral nutrition, infants, 645

Fatty acid

long-chain, disorders of propionate metabolism, 147

metabolism, biology of, mice, 38

$\mathrm{n}-3$ polyunsaturated, oxygen toxicity and, neonatal rat, 399

synthesis, high carbohydrate diet effect, 63

Fc receptor, heterogeneity, neonatal neutrophils, 6

Feeding, gastric drip, glycogen storage disease type I, 327

Fetus

atrial natriuretic factor infusion renal hemodynamic response, sheep, 291

right and left atria, rat, 339

corticosteroid and surfactant treatment, ventilated rabbit, 32

ductus arteriosus

indomethacin and betamethasone combined effect, rat, 69

ligation, neonatal pulmonary hypertension and, lamb, 239

ethanol and acetaldehyde effect, rat, 102

galactose metabolism, hepatic, rat, 161

gonadotropin and testosterone release, effect of luteinizing hormone-releasing factor agonist, sheep, 347

growth-retarded, placental calcium clearance, rat, 420

hyperphenylalaninemia-exposed, effect of large neutral amino acids, rat, 568

intrauterine growth retardation, epidermal growth factor binding, rat, 214

liver metabolism, after umbilical cord compression, 228

lung

maturation, maternal diabetes and, mouse, 173

ontogeny of epidermal growth factor receptor/kinase, 535

vitamin K-dependent carboxylase activity, rat, 530

lung surfactant phospholipid synthesis, glucocorticoid role, rat lung, 239

metabolism, maternal sodium $\beta$-hydroxybutyrate infusion and, baboon, 435

pituitary gland, acetylated and shortened endorphins in, 652

placental permeability, ${ }^{35} \mathrm{~S}$ recombinant erythropoeitin injection, sheep, 649

regulation of placental glucose, sheep, 429

response to vasoactive compounds, intrapulmonary arteries, lamb, 360

Fluid homeostasis, effects of aldosterone and atrial natriuretic peptide, neonate, 591

Flunarizine, prophylactic treatment, hypoxic-ischemic encephalopathy, rat, 573

Forskolin, myocardial inotropic effects, comparison, isoproterenol, lamb, 580

Galactose, metabolism, ovarian tissue, 151

Gangliosidosis, $\mathrm{G}_{\mathrm{M} 2}, \mathrm{~B}_{1}$ variant, 89

Gastric inhibitory polypeptide, insulin response to glucose infusion and, fullterm neonates, 490

Gestation: see also Pregnancy

corticosteroid treatment and, ventilated rabbit, 32

surfactant treatment and, ventilated rabbit, 32

Glucocorticoid, growth stunting after, catch-

Glucose up gowth after fasting, rat, 373

homeostasis, hepatic, after umbilical cord compression, fetal lamb, 228

placental transfer, fetal regulation, sheep, 429

uterine uptake, late pregnancy, guinea pig, 641

utilization, during total parenteral nutrition, infants, 645

Glucose-6-phosphatase, deficiency, gastric drip feeding and, 327

Glycogen storage disease, type I, gastric drip feedings and, 327

Gonadotropin, fetal and neonatal secretion, effect of luteinizing hormone-releasing factor agonist, sheep, 347
GP-2, reserpine treatment effect, cystic fibrosis, rat model, 478

Growth and development

after cranial irradiation, 561

catch-up growth after fasting, growthstunted rats, 373

dietary protein restriction and, rat, 509

energy requirements during catch-up growth, prediction model, 503

epidermal growth factor binding, intrauterine growth retardation, rat, 214

ethanol and acetaldehyde effect, fetal rat, 102

human milk protein intake, very low birth weight infants, 414

immature airway, in vivo mechanical properties, lamb, 143

insulin-like growth factor I binding

erythrocytes, normal prepubertal children, 409

normal and low birth weight infants, 336

intestinal maturation

mitochondrial calcium transport, rat, 107

mitochondrial phosphate transport, rat, 605

intrauterine growth retardation

adrenocortical function, term infants, 115

sympathoadrenal response to hypoxia after, neonatal rat, 466

lung function and, estimation, spatial relationship, 316

lung maturation, maternal diabetes and, fetal mouse, 173

placental calcium clearance, growth-retarded rat fetus, 420

prenatal terbutaline exposure and, lung and liver cells, neonatal rat, 617

renal $\alpha$-vasoconstriction, regional variation, sheep, 461

role of somatomedin- $\mathrm{C}$ and growth hormone, bovine growth plate chondrocyte, 76

zinc and copper deficiency, children and adolescents, 323

Growth hormone

deficiency, after cranial irradiation, 561

role in skeletal development, bovine growth plate chondrocyte, 76

Growth retardation, intrauterine, sympathoadrenal response to hypoxia after. neonatal rat, 466

Heart, atrial natriuretic factor activity, perinatal period, rat, 339

Hemorheology, neonatal, comparison, adult blood, 457

Heparin, anticoagulant effect, neonatal plasma, 405

Hepatocyte, copper toxicity and lipid peroxidation, vitamin $E$ effect, rat, 55

Histidine, transport, placental microvillous membrane vesicles, 19

Hypercapnia, cerebral vascular responses, ischemia effect, piglet, 180

Hyperinsulinemia, euglycemic, hepatic and muscle metabolism and, neonatal dog, 124

Hyperphenylalaninemia, in utero, effect of branched chain amino acids, rat, 568

Hypertension

pulmonary

after prenatal ductus arteriosus ligation, lamb, 239

calcium infusion, cardiopulmonary effects, neonate, 262 


\section{SUBJECT INDEX TO VOLUME 25}

Hyperventilation, effect on surfactant metabolism, neonatal lamb, 83

Hypocalcemia, pulmonary hypertension and, calcium infusion effects, neonate, 262

Hypoglycemia

maternal, fetal glucose production during, sheep, 429

nonketotic, acyl-coenzyme A dehydrogenase deficiency, mice, 38

Hypoxemia

arousal response, carotid-denervation and, lamb, 473

effect on myocardial blood flow, during exercise, dog, 280

myocardial oxygen consumption during, $\beta$-adrenergic influence, neonatal lamb, 585

Hypoxia

alveolar, cardiovascular response, chloralose anesthesia, neonatal lamb, 389

effect on ventilatory control, during exercise, 285

pulmonary vasoconstriction induced by, alkalosis effect, neonatal rabbit, 383

sympathoadrenal response, after intrauterine growth retardation, rat, 466

Hypoxia-ischemia, flunarizine pretreatment, infant rat, 573

Ileum, chloride-bicarbonate exchange, maturation, rat, 189

Immune factors, urinary, human milk effect, low birth weight infants, 184

Immune globulin intravenous, effect on bacterial opsonization, premature serum, 634

Immunoglobulin, production, human colostral lymphocytes, 295

Indomethacin, betamethasone and, combined effect, ductus arteriosus, fetal rat. 69

Infant: se' also Neonate

breast-fed, vitamin D metabolism, 623

diarrhea. Klebsiella pneumoniae, heat-stable enterotoxin produced by, 514

energy substrate utilization, during total parenteral nutrition, infants, 645

hypoxic-ischemic encephalopathy, flunarizine pretreatment, rat, 573

low birth weight

insulin-like growth factor-I and thyroxine relationship, 336

protein turnover, 167

urinary immune factors, human milk effect, 184

premature, periodic breathing cycle duration, 258

pulmonary oxygen toxicity, dexamethasone effect, mouse, 353

small-for-gestational-age term, adrenocortical steroids in, 115

very low birth weight

calcium and magnesium absorption from fortified human milk, 496

growth effect, human milk protein, 414

Insulin, resistance, assessment, neonatal dog, 219

Insulin-like growth factor I

binding, erythrocytes, normal prepubertal children, 409

role in skeletal development, bovine growth plate chondrocyte, 76

thyroxine and, low birth weight infants, 336

Interferon, role, Listeria monocytogenes infection, neonatal rat, 311
Interleukin-1, gestational age effect, cord blood monocytes, 342

Intestine

maturation, mitochondrial calcium transport, rat, 107

mitochondria phosphate transport, rat, 605

oxidant-producing enzymes and antioxidants in, developmental biology, piglet, 612

Iron, -sulfur clusters, deficiency, mitochondrial encephalomyopathy, 194

Iron dextran, vascular to alveolar leak, ventilated lung, immature rabbit, 130

Irradiation, cranial, growth and endocrine disorders after, 561

Ischemia, cerebral, effect on hypercapnia and acetylcholine response, piglet, 180

L-Isoleucine, metabolism, L-allo isoleucine and, maple syrup urine disease, 11

Isoproterenol, myocardial inotropic effects, comparison, forskolin, lamb, 580

Ketosis, fetal metabolism and, effect of maternal sodium $\beta$-hydroxybutyrate infusion, baboon, 435

Kidney

failure, chronic, dietary protein restriction, rat, 509

function, dietary protein restriction and, rat, 509

hemodynamic response to atrial natriuretic factor, fetal and newborn sheep, 291

$\alpha$-vasoconstriction, regional variation, sheep, 461

Killer cell, lymphokine-activated, cytotoxicity, neonatal mononuclear cells, 156

Klebsiella pneumoniae, heat-stable enterotoxin produced by, secretory diarrhea, 514

Lactation, fatty acid synthesis during, high carbohydrate diet effect, 63

Learning, verbal and spatial processing, sex chromosome abnormalities and, 577

Leukocyte

fungicidal activity, vitamin $\mathrm{D}$ resistance and, 276

polymorphonuclear

colostral antioxidant heterogeneity and, 266

nonmotile, characterization, neonates

Lipid and adults, 519

peroxidation, vitamin $\mathrm{E}$ effect, rat hepatocyte, 55

plasma, gastric drip feeding and, glycogen storage disease, 327

Lipocortin-1, ontogeny, fetal lamb lung, 535

Lipopolysaccharide, role, Listeria monocytogenes infection, neonatal rat, 311

Lipoprotein, effect on membrane fluidity, Duchenne muscular dystrophy, 656

Listeria monocytogenes, lipopolysaccharide and interferon role, neonatal rat, 311

Listeriosis, neonatal, role of lipopolysaccharides and interferons, 311

Liver

blood flow, changes at birth, sheep, 300

cellular development, prenatal terbutaline effect, neonatal rat, 617

galactose-metabolizing enzymes, pregnant rat and fetus, 161

glucose homeostasis, after umbilical cord compression, fetal lamb, 228

L-pipecolic acid oxidation, Zellweger syn- drome, 548

metabolism, euglycemic hyperinsulinemia effect, neonatal dog, 124

oxygen consumption, changes at birth, sheep, 300

palmitic acid oxidation, ammonium chloride, salicylate, and carnitine effects, rat, 119

Lung

bronchoalveolar lavage, oxygen exposure effect, rat, 396

cellular development, prenatal terbutaline effect, neonatal rat, 617

disease, acute and chronic, preterm rat model, 525

function, somatic growth and, spatial relationship estimation, 316

intrapulmonary arteries, response to vasoactive compounds, lamb, 360

iron dextran permeation, immature ventilated rabbit, 130

maturation, maternal diabetes and, fetal mouse, 173

mechanics, after surfactant replacement therapy, preterm infants, 305

ontogeny of epidermal growth factor receptor/kinase, fetal lamb, 535

oxygen toxicity, dexamethasone effect, infant mice, 353

surfactant synthesis, glucocorticoid role, fetal rat, 239

vascular pressure, patent ductus arteriosus, neonatal lamb, 136

vasculature, after prenatal ductus arteriosus ligation, lamb, 251

vasoconstriction, alkalosis effect, neonatal rabbit, 383

vitamin K-dependent carboxylase activity, fetal rat, 530

Luteinizing hormone-releasing factor, agonist, pituitary-gonadal function and, ovine fetus, 347

Lymphocyte, human colostral, immunoglobulin production by, 295

Magnesium, absorption, fortified human milk, very low birth weight infants, 496

Magnetic resonance spectroscopy

phosphorus

hypoxic-ischemic brain injury assessed by, 445

metabolite concentration ratios, neonatal brain, 440

Malnutrition, growth failure secondary to, prediction of energy requirements during catch-up growth, 503

Maple syrup urine disease, L-isoleucine and L-allo-leucine metabolism, 11

Medical education, training pediatric scientists, 1

Membrane fluidity, lipoprotein effect, Duchenne muscular dystrophy, 656

Menhaden oil, oxygen toxicity and, neonatal rat, 399

Mental retardation, maternal phenylketonuria-acquired, effect of branched chain amino acids, rat, 568

Methionine, uptake, bilirubin effect, neuro-

Milk blastoma cell line, 364

human

chemokinetic agents for monocytes in, 629

fortified, calcium and magnesium absorption, very low birth weight infants, 496 
protein intake, very low birth weight infants, 414

saccharolactone effect on bilirubin absorption and, rat, 234

urinary immune factors and, low birth weight infants, 184

vitamin $D$ metabolism, infants and mothers, 623

Milk lipid, composition, high carbohydrate diet effect, 63

Mitochondria

calcium transport, intestinal maturation and, rat, 107

encephalomyopathy, complex I deficiency in, 194

function, bilirubin effect, neuroblastoma cell line, 364

phosphate transport, rat intestine, 605

Monocytes

chemokinetic agents for, human milk, 629

cord blood, tumor necrosis factor and interleukin-1 secretion, 342

Mucin, serum levels, cystic fibrosis, 49

Muscle

contraction, intracellular calcium and, rabbit stomach, 600

metabolism, euglycemic hyperinsulinemia effect, neonatal dog, 124

Muscular dystrophy, Duchenne, membrane fluidity, lipoprotein effect, 656

Myocardium

comparison, forskolin and isoproterenol effects, young lamb, 580

oxygen consumption, $\beta$-adrenergic influence, during hypoxemia, neonatal lamb, 585

Necrotizing enterocolitis, role of reactive oxygen metabolites, piglet intestine, 612

Neonate

acute and chronic lung disease, preterm rat model, 525

anemia, prevention, method evaluation, rabbit, 274

atrial natriuretic factor infusion

renal hemodynamic response, sheep, 291

right and left atria, rat, 339

basal hemodynamics, effect of chloralose anesthesia, lamb, 389

blood, rheologic properties, 457

blood brain barrier, permeability for ${ }^{125}$ albumin-bound bilirubin, piglet, 452

blood flow, effect of arterial $\mathrm{PCO}_{2}$ variations, piglet, 205

blood viscosity, Fahraeus-Lindqvist effect, 595

brain, phosphorus metabolites and intracellular $\mathrm{pH}, 440$

cartilage cell column development, costochondral junction, 202

fatal lactic acidosis, complex III defect, 553

fluid homeostasis, aldosterone and atrial natriuretic peptide effects, 591

full-term, functional enteroinsular axis in, 490

gonadotropin and testosterone release, effect of luteinizing hormone-releasing factor agonist, sheep, 347

hepatic and muscle metabolism, euglycemic hyperinsulinemia effect, dog, 124

hyperventilation, surfactant metabolism during, lamb, 83

hypoxemia, $\beta$-adrenergic influence on myocardial oxygen consumption, 585 hypoxia, sympathoadrenal response, rat, 466

hypoxic-ischemic brain injury, assessment, phosphorus magnetic resonance spectroscopy, 445

insulin resistance, assessment, dog, 219

Listeria monocytogenes infection, lipopolysaccharide and interferon role, rat, 311

mononuclear cells, lymphokine-activated killer cytotoxicity in, 156

nonmotile neutrophil subpopulation, characterization, 519

oxygen toxicity, menhaden oil effect, rat, 399

patent ductus arteriosus, pulmonary blood pressure and, lamb, 136

plasma, anticoagulant effects of heparin, 405

polymorphonuclear neutrophil, heterogeneity, 6

pulmonary hypertension

after prenatal ductus arteriosus ligation, lamb, 239, 251

calcium infusion, cardiopulmonary effects, 262

pulmonary vasoconstriction, alkalosis effect, rabbit, 383

response to vasoactive compounds, intrapulmonary arteries, lamb, 360

seizure, diazepam effect, dog, 27

toxigenic diarrhea, cholera-toxin activated cyclase response, rat, 225

tumor necrosis factor and interleukin-1 secretion, cord blood monocytes, 342

Neuroblastoma cell, bilirubin toxicity, 364 delayed effects, 369

Neutrophil, nonmotile subpopulations, characterization, neonates and adults, 519

Nitrogen, tracer study, protein metabolism, preterm infants, 167

Norepinephrine, pulmonary artery response to, fetal and neonatal lamb, 360

Nutrition, human milk protein intake, very low birth weight infants, 414

Nutritional support, energy substrate utilization during total parenteral nutrition, infants, 645

Ornithine decarboxylase, prenatal terbutaline exposure and, lung and liver cells, neonatal rat, 617

Ovary, galactose metabolism, 151

Oxidant, injury, endothelial cell adenosine

Oxygen triphosphate depletion after, 97

consumption, changes at birth, ovine liver, 300

effect on adenosine triphosphate content, bronchoalveolar fluid, rat, 396

exposure, premature rat model, 525

toxicity

dexamethasone effect, infant mice, 353

menhaden oil effect, neonatal rat, 399

Palmitic acid, oxidation, ammonium chloride, salicylate, and carnitine effects, rat liver, 119

Pancreas

exocrine, reserpine treatment effect, cystic fiboris, rat model, 482

reserpine treatment effect, rat, 478

Parathyroid hormone, phosphate transport and, placenta, 15

Parenteral nutrition, total, energy substrate utilization, infants, 645

Patent ductus arteriosus closure, after surfactant replacerment therapy, preterm infants, 305

indomethacin and betamethasone combined effect, fetal rat, 69

pulmonary blood pressure and, neonatal lamb, 136

Pediatrics, research training, 1

Pertussis, second messenger cAMP formation in children with, 209

$\mathrm{pH}$, intracellular, magnetic resonance spectroscopy measurement, neonata brain, 440

Phenotype, psychologic, sex chromosome abnormalities and, 577

Phenyketonuria, maternal, treated with large neutral amino acids, rat, 568

Phosphate

mitochondrial transport, rat intestine, 605

placental transport, parathyroid hormone effect, 15

Phospholipid, synthesis, glucocorticoid receptor blockade and, fetal rat lung, 239

L-Pipecolic acid, oxidation, liver, Zellweger syndrome patients, 548

Pituitary, endorphin presence, human fetus, 652

Placenta

calcium clearance, growth-retarded rat fetus, 420

microvillous membrane vesicles, histidine transport by, 19

permeability, ${ }^{35} \mathrm{~S}$ recombinant erythropoietin, sheep, 649

phosphate transport, parathyroid hormone effect, 15

Polymorphonuclear neutrophil, chemotaxis and adherence, heterogeneity, neonate, 6

Potassium, influx, bilirubin effect, neuroblastoma cell line, 364

Potassium chloride, pulmonary artery response to, fetal and neonatal lamb, 360

Pregnancy

body water estimation, dilution studies, baboon, 377

galactose-metabolizing enzyme activity during, rat liver, 16

late

ethanol and acetaldehyde disposition, rat, 102

uterine glucose uptake, guinea pig, 641

maternal hypoglycemia, fetal glucose production during sheep, 429

sodium $\beta$-hydroxybutyrate infusion during, placental transfer and fetal effects, baboon, 435

Prekeratin, high molecular weight, characterization, perinatal rat epidermis, 542

Prematurity

bacterial opsonization, effect of immune globulin intravenous, 634

immature airway, in vivo mechanical properties, lamb, 143

patent ductus arteriosus, indomethacin and betamethasone combined effect, fetal rat, 69

retinopathy of, effect of arterial $\mathrm{PCO}_{2}$ variations, piglet, 205

surfactant replacement therapy, ductus arteriosus closure after, preterm infants, 305

surfactant treatment, recovery, lungs, rabbit, 423

Propionate, metabolism disorder, long-chain

Protein 


\section{SUBJECT INDEX TO VOLUME 25}

dietary restriction, effect on growth and renal function, rat, 509

human milk, growth effect, very low birth weight infants, 414

structural, perinatal rat epidermis, 542

turnover, preterm infants, 167

Puberty, alterations, after cranial irradiation, 561

Pulmonary artery, vascular pressure, patent ductus arteriosus, neonatal lamb, 136

Renin, processing, androgen influence, submandibular gland, mouse, 332

Reserpine

effect on exocrine pancreas, cystic fibrosis, rat model, 482

pancreatic growth and, cystic fibrosis, rat model, 478

Respiration, periodic breathing cycle, duration, premature infants, 258

Respiratory chain, complex III defect, fatal lactic acidosis, neonate, 553

Respiratory distress syndrome, surfactant treatment, recovery, lungs, premature rabbit, 423

Saccharolactone, bilirubin absorption and, in presence of human breast milk, 234

Salicylate, palmitic acid oxidation and, rat liver, 119

Serotonin, pulmonary artery response to, fetal and neonatal lamb, 360

Sex chromosome, abnormality, verbal and spatial processing in children with, 577

Shunt

aortopulmonary, left to right, nitroprusside effect, lamb, 44

atrial, surfactant metabolism and, neonatal lamb, 83

Sodium $\beta$-hydroxybutyrate, maternal infusion, placental transfer and fetal ef- fects, baboon, 435

Sodium nitroprusside, circulatory effects, aortopulmonary shunt, lamb, 44

Somatomedin-C, role in skeletal development, bovine growth plate chondrocyte, 76

Status epilepticus, diazepam treatment, nuclear magnetic resonance spectroscopy, dog, 27

Steroids, adrenocortical, small-for-gestational-age term infants, 115

Stomach, gastric smooth muscle, calcium channel development, rabbit, 600

Submandibular gland, renin processing, androgen influence, mouse, 332

Sudden infant death syndrome, periodic breathing cycle duration and, premature infants, 258

Surfactant

gestational effects, ventilated rabbit, 32

metabolism, during hyperventilation, neonatal lamb, 83

recovery, mechanically ventilated premature rabbit, 423

replacement therapy, ductus arteriosus closure after, premature infants, 305

Terbutaline, prenatal exposure, effect on cellular development, lung and liver, neonatal rat, 617

Testosterone, fetal and neonatal secretion, effect of luteinizing hormone-releasing factor agonist, sheep, 347

Thrombin, heparin effect, neonatal plasma, 405

Thymidine, uptake, bilirubin effect, neuroblastoma cell line, 364

Thyroxine, insulin-like growth factor-I and, low birth weight infants, 336

Triiodothyronine, effect on vitamin K-dependent carboxylation, fetal rat lung, 530
Tumor, cell lines, lymphokine-activated killer cytotoxicity against, 156

Tumor necrosis factor, gestational age effect, cord blood monocytes, 342

Tumor necrosis factor- $\alpha$, role as chemokinetic agent, monocytes, human milk, 629

Ultrasonography, artery wall properties assessed by, 94

Umbilical cord, acute compression, liver metabolism after, fetal lamb, 228

Umbilical vein, oxidant injury, endothelial cell adenosine triphosphate depletion after, 97

Uterus, glucose uptake, late pregnancy, guinea pig, 641

Vasoconstriction, renal, postjunctional adrenoceptor responses, sheep, 461

Vitamin D

hereditary resistance, defective leukocyte fungicidal activity, 276

metabolism, breast-fed infants and their mothers, 623

1,25- $(\mathrm{OH})_{2}$-Vitamin $\mathrm{D}_{3}$, mitochondrial phosphate transport regulated by, rat intestine, 605

Vitamin E

copper toxicity and, isolated hepatocyte, rat, 55

lipid peroxidation and, isolated hepatocyte, rat, 55

Water, doubly labeled, energy requirements predicted by, malnourished children, 503

Zellweger syndrome, L-pipecolic acid oxidation in, 548

Zinc, deficiency, growth status and, 323 